\title{
Remifentanil Associado ao Propofol ou Sevoflurano para Colecistectomia Videolaparoscópica. Estudo Comparativo *
}

\author{
Remifentanil Associated to Propofol or Sevoflurane for \\ Videolaparoscopic Cholecystectomy. A Comparative Study
}

\author{
Fernanda Fischer Estivalet ${ }^{1}$, Airton Bagatini, TSA ${ }^{2}$, Cláudio Roberto Gomes ${ }^{3}$
}

\section{RESUMO}

Estivalet FF, Bagatini A, Gomes CR - Remifentanil Associado ao Propofol ou Sevoflurano para Colecistectomia Videolaparoscópica. Estudo Comparativo

Justificativa e Objetivos - Técnicas de anestesia venosa, inalatória ou combinadas têm sido utilizadas para colecistectomias por videolaparoscopia. O objetivo deste estudo foi comparar o emprego de remifentanil associado ao propofol ou sevoflurano em relação aos parâmetros hemodinâmicos, a recuperação da anestesia e aos efeitos colaterais.

Método - Foram estudados 40 pacientes, divididos em 2 grupos: Grupo 1 (G1) - Indução com propofol em concentração plasmática alvo-controlada de $3 \mu \mathrm{g} \cdot \mathrm{ml}^{-1}$ e remifentanil na dose de $0,3 \mu \mathrm{g} \cdot \mathrm{kg}^{-1} \cdot \mathrm{min}^{-1} \mathrm{em}$ bomba de infusão. Atracúrio $(0,5$ $\mathrm{mg} \cdot \mathrm{kg}^{-1}$ ) foi administrado para a intubação traqueal. A anestesia foi mantida com propofol em infusão alvo-controlada de 2,5 a $3 \mu \mathrm{g} \cdot \mathrm{ml}^{-1}$ e oxigênio a $100 \%$, e remifentanil em infusão contínua $\left(0,3 \mu \mathrm{g} \cdot \mathrm{kg}^{-1} \cdot \mathrm{min}^{-1}\right)$. Grupo 2 (G2) - Indução com sevoflurano na concentração de $6 \%$ e oxigênio a $100 \% \mathrm{com}$ fluxo de 4 L. $\mathrm{min}^{-1}$ e remifentanil na dose de $0,3 \mu \mathrm{g} \cdot \mathrm{kg}^{-1} \cdot \mathrm{min}^{-1}$, em bomba de infusão. Após a perda da consciência, a concentração de sevoflurano foi reduzida para $2 \%$ com fluxo de oxigênio de 2 L. $\mathrm{min}^{-1}$; da mesma forma que o G1, foi administrado atracúrio na dose de $0,5 \mathrm{mg} \cdot \mathrm{kg}^{-1}$. A infusão de remifentanil foi mantida durante toda a cirurgia na dose de 0,3 $\mu \mathrm{g} \cdot \mathrm{kg}^{-1} \cdot \mathrm{min}^{-1}$. A média da freqüência cardíaca (FC), e das pressões arteriais sistólica (PAS), diastólica (PAD) e média (PAM) foram medidas nos seguintes momentos: $M 1$, antes da indução anestésica; M2, 1 minuto antes da IOT; M3, 1 minuto após IOT; M4, 5 minutos após IOT; M5, 5 minutos após a incisão cirúrgica; M6 e M7,10 e 30 minutos, respectivamente, após o pneumoperitônio. Após o término do procedimento foram analisados os tempos, em minutos, para abertura ocular, ventilação espontânea, extubação, fala do nome completo e o apertar de mão do anestesiologista, sob comando. Também foram estudadas a freqüência do uso de atropina e efedrina e a ocorrência de náuseas, vômitos e dor.

Resultados - As alterações hemodinâmicas (PAS, PAD, PAM e $F C)$ e o despertar dos pacientes não tiveram diferenças significativas entre os grupos. O uso de efedrina e atropina

\footnotetext{
* Recebido do (Received from) Centro de Ensino e Treinamento em Anestesiologia do SANE - Porto Alegre, RS

1. $M E_{2}$ do $C E T / S B A$ do SANE

2. Co-responsável pelo CET/SBA do SANE

3. Anestesiologista do Hospital Ernesto Dornelles
}

Apresentado (Submitted) em 15 de agosto de 2001

Aceito (Accepted) para publicação em 06 de dezembro de 2001

Correspondência para (Mail to):

Dr. Airton Bagatini

Rua Santana, 483/301

90040-373 Porto Alegre, RS

E-mail: airtonb@matrix.com.br

(c) Sociedade Brasileira de Anestesiologia, 2002 foram semelhantes. Entre as complicações somente os vômitos tiveram maior incidência no Grupo 2.

Conclusões - Ambas as técnicas promoveram diminuição da pressão arterial e da freqüência cardíaca. A recuperação anestésica é semelhante para as duas técnicas. A incidência de vômitos é maior quando o remifentanil foi associado ao sevoflurano.

UNITERMOS - ANALGÉSICOS, Opióides: remifentanil; ANESTÉSICOS, Inalatório: sevoflurano; HIPNÓTICOS: propofol

\section{SUMMARY}

Estivalet FF, Bagatini A, Gomes CR - Remifentanil Associated to Propofol or Sevoflurane for Videolaparoscopic Cholecystectomy. A Comparative Study

Background and Objectives - Different intravenous, inhalational or combined anesthetic techniques have been used for videolaparoscopic cholecystectomy. This study aimed at comparing hemodynamic parameters, anesthetic recovery and side-effects of remifentanil associated to propofol or sevoflurane.

Methods - Participated in this study 40 patients, who were divided in two groups: Group 1 (G1) - induced with $3 \mu \mathrm{g} \cdot \mathrm{ml}^{-1}$ propofol and a $0.3 \mu \mathrm{g} \cdot \mathrm{kg}^{-1} \cdot \mathrm{min}^{-1}$ remifentanil infusion. Atracurium (0.5 mg. $\mathrm{kg}^{-1}$ ) was administered for tracheal intubation. Anesthesia was maintained with 2.5 to $3 \mu \mathrm{g} \cdot \mathrm{ml}^{-1}$ propofol in target controlled infusion and $100 \%$ oxygen, and remifentanil by continuous infusion $\left(0.3 \mu \mathrm{g} \cdot \mathrm{kg}^{-1} \cdot \mathrm{min}^{-1}\right)$; Group 2 (G2) - induced with $6 \%$ sevoflurane and $100 \%$ oxygen at a 4 L. min ${ }^{-1}$ flow and a $0.3 \mu \mathrm{g} \cdot \mathrm{kg}^{-1}$. $\mathrm{min}^{-1}$ remifentanil infusion. After loss of consciousness, sevoflurane concentration was decreased to $2 \%$ with a 2 L. min $^{-1}$ oxygen flow. Similarly to G1, 0.5 $\mathrm{mg} \cdot \mathrm{kg}^{-1}$ atracurium was administered. A $0.3 \mu \mathrm{g} \cdot \mathrm{kg}^{-1} \cdot \mathrm{min}^{-1}$ remifentanil infusion was maintained throughout the surgery. Mean heart rate (HR) and systolic (SBP) and diastolic (DBP) blood pressure were measured in the following moments: M1, before anesthetic induction; M2, 1 minute before TI; M3, 1 minute after TI; M4, 5 minutes after TI; M5, 5 minutes after incision; M6 and M7, 10 and 30 minutes after pneumoperitoneum, respectively. After surgery, the time for eye opening, spontaneous ventilation, extubation, saying the complete name and anesthesiologist's hand shaking under verbal command were measured in minutes: Atropine and ephedrine requirements, incidence of nausea, vomiting and pain were also evaluated.

Results - Hemodynamic changes (SBP, DBP, MBP and HR) and patients' emergence time were not significantly different between groups. There were similar ephedrine and atropine requirements. Among complications only vomiting was higher incidence in group 2.

Conclusions - Both techniques decrease blood pressure and heart rate. Anesthetic recovery was similar for both techniques. A higher incidence of vomiting was observed when remifentanil was associated to sevoflurane.

KEY WORDS - ANALGESICS: Opioids: remifentanil; ANESTHETICS, Inhalational: sevoflurane; HIPNOTICS: propofol 


\section{INTRODUÇÃO}

A indução anestésica com propofol é mais rápida que a indução inalatória em adultos, mesmo quando são usados os agentes voláteis mais recentes com baixos coeficientes de partição sangue-gás. A indução com propofol produz menor irritação das vias aéreas quando comparado com o desflurano e sem diferença quando comparado ao sevoflurano. Estudo mostra que os tempos de recuperação após o uso de propofol, associado ao óxido nitroso para indução e manutenção foram semelhantes aos obtidos com o sevoflurano associado ao $\mathrm{N}_{2} \mathrm{O}^{1}$.

Para colecistectomias laparoscópicas, Blobner e col. ${ }^{2}$ relataram que uma técnica de anestesia venosa total (AVT) com propofol como droga principal, não ofereceu vantagens em relação à anestesia balanceada com isoflurano.

O sevoflurano mostrou-se eficaz quando utilizado como principal anestésico em cirurgias videolaparoscópicas. Destaca-se a estabilidade da freqüência cardíaca associada a um despertar rápido e baixa incidência de efeitos colaterais no per e pós-operatório imediato ${ }^{3}$.

O remifentanil é um agonista opióide sintético estruturalmente relacionado ao fentanil, mas é ímpar entre os opióides por causa de sua ligação éster, que o torna suscetívelà hidrólise rápida pelas esterases inespecíficas do sangue e tecidos até metabólitos inativos. Ele é o primeiro opióide de ação ultra-curta verdadeira para uso como complemento à anestesia geral, venosa ou inalatória.

O objetivo deste estudo é comparar duas técnicas anestésicas utilizando remifentanil em infusão contínua, associado ao propofol ou ao sevoflurano, em pacientes submetidos a colecistectomias videolaparoscópicas.

\section{MÉTODO}

Após a aprovação da Comissão de Ética, participaram do estudo 40 pacientes adultos de ambos os sexos, estado físico ASA I ou II submetidos à colecistectomia por via laparoscópica. Foram excluídos do estudo os pacientes que não receberam medicação pré-anestésica e cirurgias convertidas para o método convencional (cirurgia aberta).

Todos os pacientes receberam lorazepam $(2 \mathrm{mg})$ como medicação pré-anestésica, por via oral, 1 h e 30 minutos antes do procedimento. Na sala operatória, foi feita monitorização com cardioscópio na derivação $D_{\|}$, oxímetro de pulso, pressão não-invasiva e, após a intubação traqueal, com capnografia e analisador de gases.

Após venóclise, realizada com cateter venoso 18 ou 20G, foram administrados $500 \mathrm{ml}$ de solução fisiológica a $0,9 \%$. Antes da indução anestésica, foram administrados cetoprofeno (100 mg)e dipirona $(1 \mathrm{~g})$ para analgesia pós-operatória. Após pré-oxigenação, a indução e a manutenção anestésica foram feitas da seguinte forma: Grupo 1 (G1) - Indução com propofol em concentração plasmática alvo-controlada de 3 $\mu \mathrm{g} \cdot \mathrm{ml}^{-1}$ e remifentanil na dose de $0,3 \mu \mathrm{g} \cdot \mathrm{kg}^{-1} \cdot \mathrm{min}^{-1} \mathrm{em}$ bomba de infusão. Após abolido o reflexo palpebral foi administrado atracúrio na dose de $0,5 \mathrm{mg} \cdot \mathrm{kg}^{-1}$ para a intubação traqueal. A anestesia foi mantida com propofol em infusão alvo-controlada de 2,5 a $3 \mu \mathrm{g} \cdot \mathrm{ml}^{-1}$ e oxigênio a $100 \%$, e remifentanil em infusão contínua $\left(0,3 \mu \mathrm{g} \cdot \mathrm{kg}^{-1} \cdot \mathrm{min}^{-1}\right)$. Grupo 2 ( G2) - Indução com sevoflurano na concentração de $6 \%$ e oxigênio a $100 \%$ com fluxo de $4 \mathrm{~L} \cdot \mathrm{min}^{-1}$ e remifentanil na dose de $0,3 \mu \mathrm{g} \cdot \mathrm{kg}^{-1} \cdot \mathrm{min}^{-1}$, em bomba de infusão. Após a perda da consciência, a concentração de sevoflurano foi reduzida para $2 \%$ com fluxo de oxigênio de 2 L. min $^{-1}$; da mesma forma que o G1, após a perda do reflexo palpebral foi administrado atracúrio na dose de 0,5 $\mathrm{mg} \cdot \mathrm{kg}^{-1}$. A infusão de remifentanil foi mantida durante toda a cirurgia na dose de $0,3 \mu \mathrm{g} \cdot \mathrm{kg}^{-1} \cdot \mathrm{min}^{-1}$. As médias da freqüência cardíaca (FC), pressão arterial sistólica (PAS), pressão arterial média (PAM) e pressão arterial diastólica (PAD) foram verificadas e registradas nos seguintes momentos: $M 1$, antes da indução anestésica; M2,1 minuto antes da intubação orotraqueal (IOT); M3, 1 minuto após IOT; M4, 5 minutos após IOT; M5, 5 minutos após incisão cirúrgica; M6 e M7, 10 e 30 minutos respectivamente após o pneumoperitônio. A análise destes dados foi feita pelo teste $t$ de Student $(p<0,05)$.

Os pacientes que apresentaram diminuição da PAS maior que $30 \%$ ou $\mathrm{FC}$ menor que $50 \mathrm{bpm}$ foram tratados com efedrina $(10 \mathrm{mg})$ e atropina $(0,5 \mathrm{mg})$, respectivamente. A cada dose administrada era anotado seu uso, sendo o resultado analisado pelo teste Exato de Fisher.

Após o término do procedimento, foi encerrada a infusão dos anestésicos e, então, foram anotados os tempos, em minutos, para abertura ocular, ventilação espontânea, extubação, a fala do nome completo e o apertar de mão do anestesiologista, sob comando. Estes dados foram analisados pelo teste $t$ de Student.

Na sala de recuperação pós-anestésica, 2 horas após o término do procedimento, os pacientes foram argüidos sobre a presença de dor, náuseas e vômitos. Os dados foram analisados pelo teste Exato de Fisher.

\section{RESULTADOS}

As características dos pacientes (sexo, idade, peso e altura) foram semelhantes entre os grupos. Também não houve diferenças significativas quanto ao estado físico dos pacientes; no entanto, apenas 8 pacientes foram classificados como ASAI ( 3 do grupo 1 e 5 do grupo 2). Amédia de tempo cirúrgico foi de 120,95 minutos para o grupo do propofol e de 118,6 minutos para o grupo do sevoflurano, não apresentando também diferenças significativas (Tabela I).

As alterações da PAS, PAD e PAM (Figuras 1, 2 e 3) foram semelhantes nos dois grupos nos momentos estudados, bem como as alterações da freqüência cardíaca (Figura 4), principalmente quanto à presença de bradicardia. A necessidade do uso de efedrina foi semelhante nos dois grupos, no entanto, no grupo 1 metade dos pacientes necessitaram do uso de efedrina, enquanto no segundo grupo $70 \%$ dos pacientes necessitaram do vasopressor, representando uma incidência um pouco maior de hipotensão arterial no grupo 2. Quanto ao uso de atropina, foi necessária a utilização em 5 pacientes do grupo 1 e em 10 pacientes do grupo 2, o que não foi estatisticamente significativo (Tabela II). 
Tabela I - Dados Demográficos

\begin{tabular}{lcc}
\hline & $\begin{array}{c}\text { Grupo 1 (Propofol) } \\
(\mathrm{n}=20)\end{array}$ & $\begin{array}{c}\text { Grupo 2 (Sevoflurano) } \\
(\mathrm{n}=20)\end{array}$ \\
\hline Idade (anos) ${ }^{*}$ & $53,65 \pm 11,21$ & $52,95 \pm 16,51$ \\
Peso $(\mathrm{kg})^{*}$ & $72,30 \pm 12,90$ & $77,85 \pm 17,19$ \\
Altura $(\mathrm{m})^{*}$ & $1,62 \pm 0,95$ & $1,63 \pm 0,11$ \\
Tempo de cirurgia (min) ${ }^{*}$ & $120,95 \pm 43,51$ & $118,60 \pm 38,57$ \\
Sexo ** & $8 / 12$ & $7 / 13$ \\
$\quad$ Masculino & 8 & 7 \\
$\quad$ Feminino & 12 & 13 \\
Estado físico (ASA) ** & & \\
I & 3 & 5 \\
II & 17 & 15 \\
\hline
\end{tabular}

* Valores expressos pela Média \pm DP (teste $t$ de Student; $p>0,05$ )

** Teste de Exato de Fisher $(p>0,05)$ (prevalência do sexo feminino)

Tabela II - Uso de Atropina e/ou Efedrina

\begin{tabular}{lcc}
\hline Droga & $\begin{array}{c}\text { Grupo 1 (Propofol) } \\
(\mathrm{n}=20)\end{array}$ & $\begin{array}{c}\text { Grupo 2 (Sevoflurano) } \\
(\mathrm{n}=20)\end{array}$ \\
\hline Atropina & 5 & $10^{*}$ \\
Efedrina & 10 & $14^{*}$ \\
\hline
\end{tabular}

* Teste Exato de Fisher $(p<0,05)$

Em relação ao tempo para abertura ocular, ventilação espontânea, extubação, apertar a mão e dizer o nome completo também não houve diferença significativa entre os grupos (Tabela III).

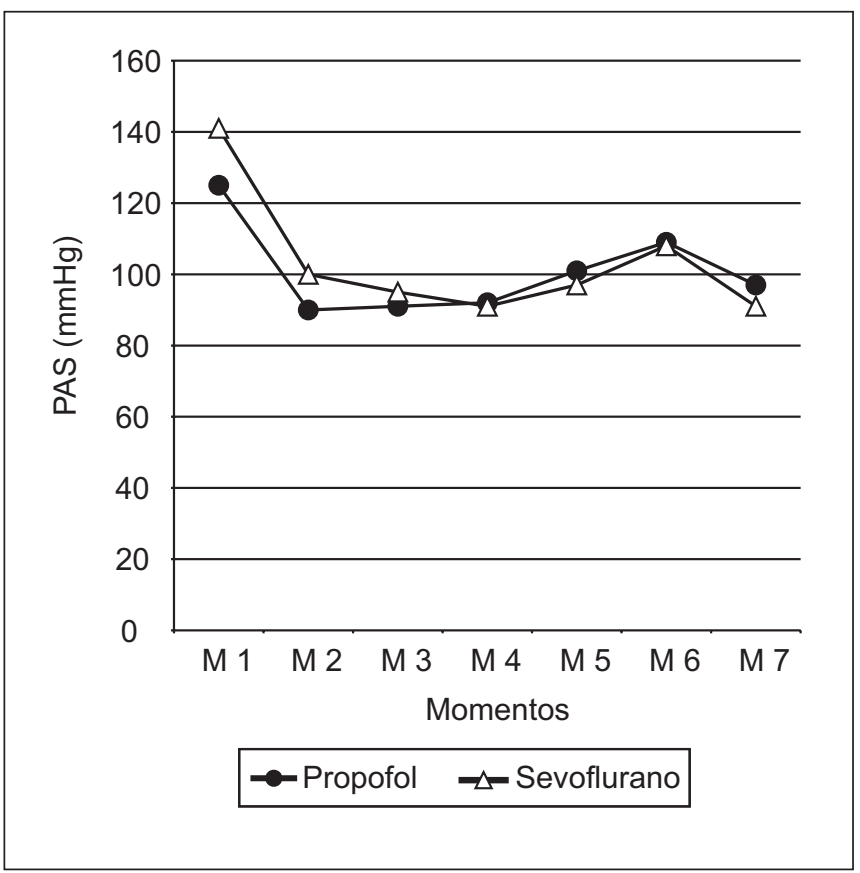

Figura 1 - Pressão Arterial Sistólica $(p>0,05)$

Revista Brasileira de Anestesiologia

Vol. 52, № 4, Julho - Agosto, 2002

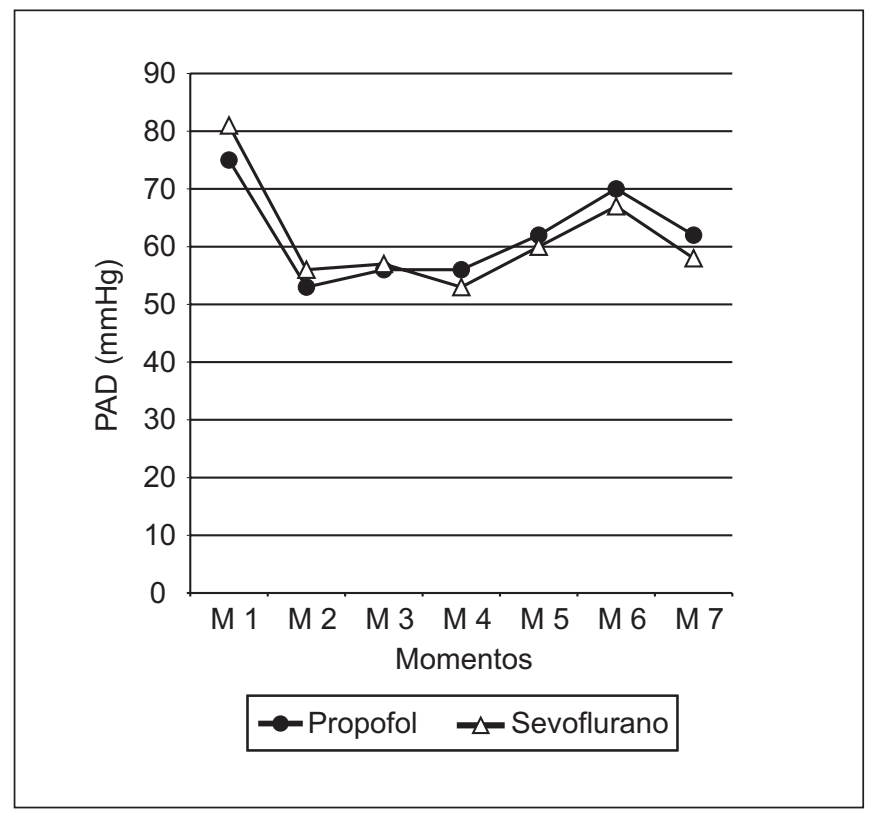

Figura 2 - Pressão Arterial Diastólica $(p>0,05)$

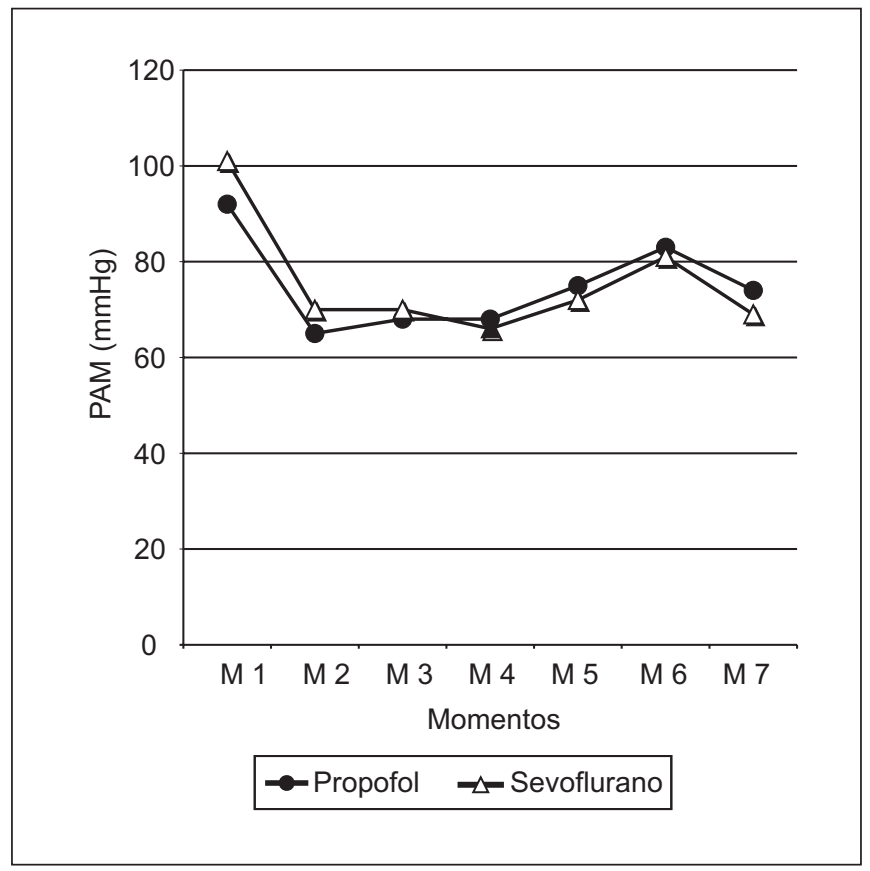

Figura 3 - Pressão Arterial Média $(p>0,05)$

Tabela III - Recuperação Pós-Anestésica (Média \pm DP em minutos)

\begin{tabular}{lcc}
\hline & $\begin{array}{c}\text { Grupo 1 (Propofol) } \\
(\mathrm{n}=20)\end{array}$ & $\begin{array}{c}\text { Grupo 2 (Sevoflurano) } \\
(\mathrm{n}=20)\end{array}$ \\
\hline Abertura ocular & $11,10 \pm 4,05$ & $14,39 \pm 6,10$ \\
Ventilação espontânea & $11,10 \pm 4,27$ & $10,74 \pm 4,22$ \\
Extubação & $11,43 \pm 4,32$ & $13,92 \pm 5,36$ \\
Apertar a mão & $12,50 \pm 4,06$ & $15,25 \pm 6,00$ \\
Dizer o nome & $12,88 \pm 3,97$ & $16,04 \pm 4,65$ \\
\hline
\end{tabular}

Teste $t$ de Student $(p>0,05)$ 


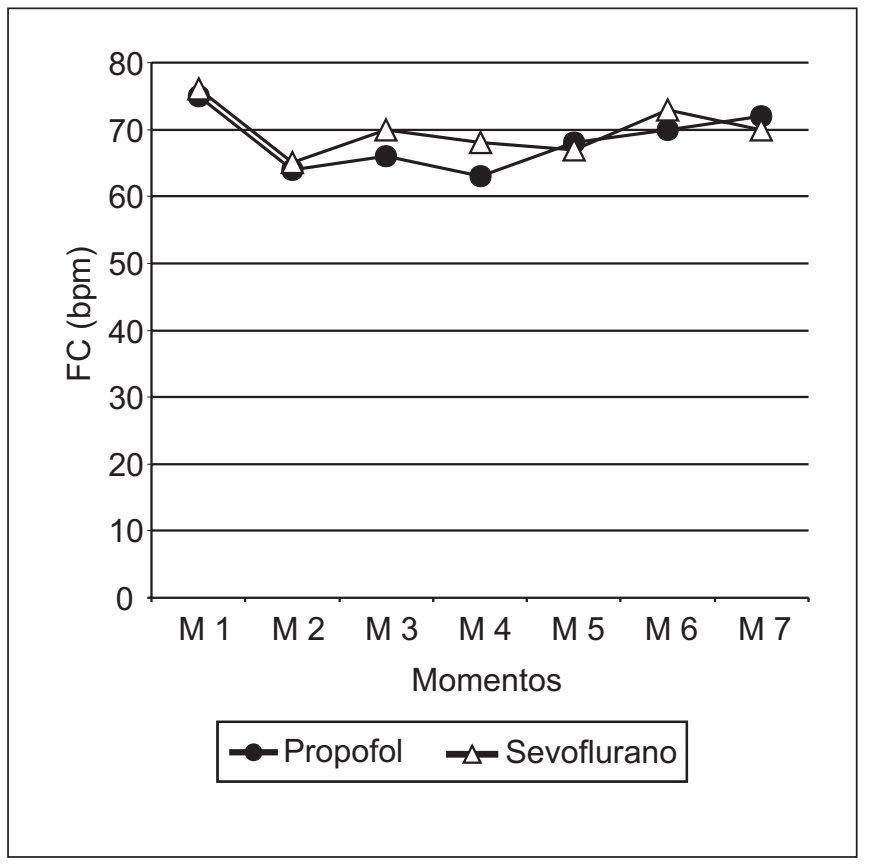

Figura 4 - Freqüência Cardíaca $(p>0,05)$

Entre as complicações pós-operatórias estudadas, a presença de náuseas e dor não foi estatisticamente significativa entre os grupos. O grupo 1 apresentou 4 pacientes que referiram náuseas, e no grupo 2, 7 pacientes relataram o sintoma. Em relação à dor, o número de pacientes foi idêntico nos dois grupos (8 pacientes em cada). Ao se tratar da incidência de vômitos houve diferença significantemente estatística entre os grupos. Onze pacientes (55\%) do grupo 2 apresentaram vômitos pós-operatórios, contra apenas 1 paciente $(5 \%)$ do grupo 1 (Tabela IV).

Tabela IV - Complicações Pós-Operatórias

\begin{tabular}{lcc}
\hline & $\begin{array}{c}\text { Grupo 1 (Propofol) } \\
(\mathrm{n}=20)\end{array}$ & $\begin{array}{c}\text { Grupo 2 (Sevoflurano) } \\
(\mathrm{n}=20)\end{array}$ \\
\hline Náusea & 4 & 7 \\
Vômitos & 1 & $11^{*}$ \\
Dor & 8 & 8 \\
\hline
\end{tabular}

* Teste Exato de Fischer $(p<0,05)$

\section{DISCUSSÃO}

Este estudo demonstrou que ambas as técnicas apresentaram resultados hemodinâmicos semelhantes para colecistectomias videolaparoscópicas, havendo diminuição das pressões arteriais e da freqüência cardíaca nos momentos estudados. Durante a indução da anestesia com propofol-remifentanil, a magnitude da hipotensão arterial e da bradicardia depende da velocidade de infusão do propofol e da dose do opióide ${ }^{4,5}$. Na indução com o sevoflurano, alguns autores ${ }^{6}$ referem menos hipotensão arterial quando comparado com o propofol. No nosso estudo a ocorrência de hipotensão arterial e a bradicardia podem estar relacionadas diretamente com a infusão do remifentanil, que por si só apresenta esses paraefeitos dose-dependentes ${ }^{7}$. Alguns estudos ${ }^{8,9}$ relatam que a mínima concentração de propofol para que o reflexo palpebral seja abolido, na maioria dos pacientes, pode ser muito alta para pacientes idosos, promovendo assim maior diminuição na PA. O remifentanil também deve ter sua dose reduzida em $50 \%$ para pacientes com idade maior que 65 anos, para que ocorra menor bradicardia e hipotensão ${ }^{10}$. É recomendada a infusão de cristalóides como prevenção ou tratamento de hipotensão arterial inicial ${ }^{11}$. Neste estudo, todos os pacientes receberam, antes da indução, $500 \mathrm{ml}$ de solução fisiológica 0,9\%. Estudos referem que a dose para indução com propofol produz diminuição de até $30 \%$ da pressão arterial, especialmente se associado com a infusão de opióides; neste estudo foi observada uma queda em metade dos pacientes do grupo 1. Esta diminuição referida, não foi associada à normalização da pressão arterial no momento da intubação traqueal, mostrando o bloqueio da resposta hipertensiva no momento da laringoscopia, como descrito em estudo comparativo entre sevoflurano-propofol e alfentanil ${ }^{12}$. Apesar da incidência relativamente alta de hipotensão arterial, neste estudo, esta foi de curta duração e facilmente contornada com uso de vasopressor.

Em relação aos parâmetros utilizados para avaliar a recuperação da anestesia (tempos para abertura ocular, ventilação espontânea, extubação, apertar a mão e dizer o nome completo), não foram encontradas diferenças estatisticamente significativas, porém, na maioria dos parâmetros avaliados o grupo do propofol apresentou tempos mais curtos. Jellish e col. ${ }^{13}$ referem o tempo de despertar mais precoce para o sevoflurano, devido ao seu perfil de rápida recuperação, proporcionado por sua pequena solubilidade e pouco acúmulo nos tecidos. Após longas infusões, a meia-vida de eliminação do propofol está aumentada, por ocorrer acúmulo da droga em tecidos periféricos pobremente vascularizados. É provável que os tempos para recuperação tenham sido semeIhantes em nosso estudo devido à infusão não-prolongada do propofol, não havendo então este acúmulo da droga ${ }^{13}$. Outros estudos ${ }^{6,14}$ também demonstraram recuperação mais rápida com o sevoflurano quando comparado com o propofol, porém não foram estatisticamente significativos. Ao avaliar a incidência de complicações pós-operatórias, como náuseas, vômitos e a presença de dor, não foi encontrada diferença significativa estatisticamente quanto a náuseas e dor. Ao se tratar de presença de vômitos houve diferença significativa estatisticamente maior no grupo do sevoflurano. Alguns trabalhos relatam que houve incidência semelhante de náuseas e vômitos pós-operatórios tanto nos pacientes que utilizaram o sevoflurano, quanto nos pacientes que utilizaram o propofol ${ }^{13-15}$. Hwan e col. ${ }^{16}$ mostraram resultados semelhantes ao encontrado no presente estudo, com menos incidência de náuseas e vômitos nos pacientes do grupo do propofol. O propofol apresenta características antieméticas, mesmo quando se trata de cirurgias que produzem náuseas e vômitos ${ }^{17}$, como no caso colecistectomias. 
Outros fatores que contribuiriam para a presença desses sintomas seriam obesidade, diabete, pacientes jovens e do sexo feminino. Neste estudo a população foi predominantemente feminina em ambos os grupos.

A presença de vômitos pós-operatórios nos pacientes do grupo 2 poderia estar relacionada com o uso do opióide, se não se tratasse de um opióide de rápida eliminação como o remifentanil. Embora o sevoflurano, por não apresentar odor desagradável e recuperação rápida das funções cognitivas, cause menos náuseas e vômitos do que os outros agentes inalatórios, ele não apresenta as características antieméticas do propofol.

A presença de dor foi idêntica em ambos os grupos neste estudo. Em alguns artigos ${ }^{13,14}$ foi relatada a necessidade mais precoce de analgesia nos pacientes que foram submetidos à anestesia com o sevoflurano, mesmo que sem diferença estatisticamente significante.

Os dados obtidos neste estudo permitem concluir que: a) ambas as técnicas promovem diminuição da pressão arterial e da freqüência cardíaca; b) a recuperação anestésica é semelhante para as duas técnicas; c) a incidência de vômitos é maior quando o remifentanil é associado ao sevoflurano do que com o propofol em pacientes submetidos à colecistectomia por via laparoscópica.

\section{Remifentanil Associated to Propofol or Sevoflurane for Videolaparoscopic Cholecystectomy. A Comparative Study}

Fernanda Fischer Estivalet, M.D., Airton Bagatini, TSA, M.D., Cláudio Roberto Gomes, M.D.

\section{INTRODUCTION}

Anesthetic induction with propofol in adults is faster than inhalational induction, even when the more recent volatile agents with lower blood-gas partition coefficients are used. Propofol induction causes less airway irritation when compared to desflurane and no difference when compared to sevoflurane. A study has shown that recovery times with propofol associated to nitrous oxide for induction and maintenance were similar to those obtained with sevoflurane associated to $\mathrm{N}_{2} \mathrm{O}{ }^{1}$.

Blobner et al. ${ }^{2}$ have reported that a total intravenous anesthesia (TIVA) with propofol as the major drug was not advantageous in laparoscopic cholecystectomies as compared to sevoflurane balanced anesthesia.

Sevoflurane was effective when used as the major anesthetic in videolaparoscopic surgeries. Heart rate stability associated to faster emergence and low incidence of perioperative or immediate postoperative side-effects are to be highlighted ${ }^{3}$. Remifentanil is a synthetic opioid agonist structurally related to fentanyl, but it is unique among the opioids for its ester linkage, which makes it susceptible to fast hydrolysis by nons- pecific esterase's of blood and tissues to inactive metabolites. It is the first true ultra short acting opioid to be used as a complement to general intravenous or inhalational anesthesia.

This study aimed at comparing two anesthetic techniques with remifentanil in continuous infusion associated to propofol or sevoflurane in patients submitted to videolaparoscopic surgery.

\section{METHODS}

After the Ethical Committee approval, participated in this study 40 adult patients of both genders, physical status ASA I or II, submitted to laparoscopic cholecystectomy. Patients not premedicated and surgeries converted to the traditional method (open surgery) were excluded from the study.

All patients were premedicated with oral lorazepam $(2 \mathrm{mg})$, 90 minutes before surgery. Monitoring in the operating room consisted of cardioscopy in $D_{\text {II }}$, pulse oximetry, non-invasive blood pressure and, after tracheal intubation, capnography and gas analysis.

After venopuncture with an 18 or $20 \mathrm{G}$ catheter, $500 \mathrm{ml}$ of $0.9 \%$ saline solution were administered. Before anesthetic induction, ketoprofen $(100 \mathrm{mg})$ and dipirone $(1 \mathrm{~g})$ were administered for postoperative analgesia.

After pre-oxygenation, anesthetic induction and maintenance were performed as follows: Group 1 (G1) - induction with 3 $\mu \mathrm{g} \cdot \mathrm{ml}^{-1}$ propofol and $0.3 \mu \mathrm{g} \cdot \mathrm{kg}^{-1} \cdot \mathrm{min}^{-1}$ remifentanil by an infusion pump. After loss of the eyelid reflex, $0.5 \mathrm{mg} . \mathrm{kg}^{-1}$ atracurium was administered for tracheal intubation. Anesthesia was maintained with 2.5 to $3 \mu \mathrm{g} \cdot \mathrm{ml}^{-1}$ propofol in target controlled infusion, $100 \%$ oxygen, and remifentanil continuous infusion $\left(0.3 \mu \mathrm{g} \cdot \mathrm{kg}^{-1} \cdot \mathrm{min}^{-1}\right)$; Group 2 (G2) - induction with $6 \%$ sevoflurane and $100 \%$ oxygen at a $4 \mathrm{~L} \cdot \mathrm{min}^{-1}$ flow and 0.3 $\mu \mathrm{g} \cdot \mathrm{kg}^{-1} \cdot \mathrm{min}^{-1}$ remifentanil in infusion pump. After loss of consciousness, sevoflurane concentration was decreased to $2 \%$ in a 2 L.min ${ }^{-1}$ oxygen flow. Similarly to $\mathrm{G} 1,0.5 \mathrm{mg} \cdot \mathrm{kg}^{-1}$ atracurium was administered after loss of the eyelid reflex. A $0.3 \mu \mathrm{g} \cdot \mathrm{kg}^{-1} \cdot \mathrm{min}^{-1}$ remifentanil infusion was maintained throughout the surgery. Mean heart rate $(\mathrm{HR})$ and systolic (SBP) and diastolic (DBP) blood pressure were measured and recorded in the following moments: $M 1$, before anesthetic induction; M2, 1 minute before TI; M3, 1 minute after TI; M4, 5 minutes after TI; M5, 5 minutes after incision; M6 and $M 7,10$ and 30 minutes after pneumoperitoneum, respectively. Data was analyzed by Student's $t$ test $(p<0.05)$.

Patients with a SBP decrease greater than $30 \%$ or HR lower than $50 \mathrm{bpm}$ were treated with ephedrine $(10 \mathrm{mg})$ and atropine $(0.5 \mathrm{mg})$, respectively. Every dose was recorded and the results were analyzed by Fisher's exact test.

After surgery, anesthetic infusion was stopped and the following parameters were measured (in minutes): time for eye opening, spontaneous ventilation, extubation, saying the complete name and anesthesiologist's hand shaking following verbal command. Data was also analyzed by Student's $t$ test. 
In the post-anesthetic recovery unit and 2 hours after surgery, patients were asked about pain, nausea and vomiting. Data was analyzed by Fisher's exact test.

\section{RESULTS}

Demographic data (gender, age, weight and height) was similar in both groups. There were also no significant differences in physical status, although only 8 patients were classified as ASAI ( 3 in group 1 and 5 in group 2). Mean surgery duration was $120.95 \mathrm{~min}$ in the propofol group and $118.6 \mathrm{~min}$ in the sevoflurane group, without statistically significant differences (Table I).

Table I - Demographics Data

\begin{tabular}{lcc}
\hline & $\begin{array}{c}\text { Group } 1 \text { (Propofol) } \\
(\mathrm{n}=20)\end{array}$ & $\begin{array}{c}\text { Group } 2 \text { (Sevoflurane) } \\
(\mathrm{n}=20)\end{array}$ \\
\hline Age (years) * & $53.65 \pm 11.21$ & $52.95 \pm 16.51$ \\
Weight $(\mathrm{kg}){ }^{*}$ & $72.30 \pm 12.90$ & $77.85 \pm 17.19$ \\
Height $(\mathrm{m}){ }^{*}$ & $1.62 \pm 0.95$ & $1.63 \pm 0.11$ \\
Surgery duration (min) * & $120.95 \pm 43.51$ & $118.60 \pm 38.57$ \\
Gender ** & $8 / 12$ & $7 / 13$ \\
$\quad$ Male & 8 & 7 \\
Female & 12 & 13 \\
Physical status (ASA) ** & & 5 \\
I & 3 & 15 \\
II & 17 & $5.05)$ \\
\hline
\end{tabular}

*Values expressed by the Mean \pm SD (Student's $t$ test $p>0.05$ )

** Fisher's Exact test $(p>0.05)$ (prevalence of females)

SBP, DBP, MBP and HR changes (Figures 1, 2 and 3) were similar for both groups (Figure 4), especially as to the presence of bradycardia. Ephedrine requirement was similar between groups, however in group 1 half the patients needed ephedrine as compared to $70 \%$ in group 2 , thus with a slightly higher incidence of arterial hypotension in group 2 . Atropine was administered to five patients in group 1 and to ten patients in the group 2, difference that was not statistically significant (Table II).

Table II - Atropine and/or Ephedrine Requirement

\begin{tabular}{lcc}
\hline Drug & $\begin{array}{c}\text { Group 1 (Propofol) } \\
(\mathrm{n}=20)\end{array}$ & $\begin{array}{c}\text { Group 2 (Sevoflurane) } \\
(\mathrm{n}=20)\end{array}$ \\
\hline Atropine & 5 & $10^{*}$ \\
Ephedrine & 10 & $14^{*}$ \\
\hline
\end{tabular}

${ }^{*}$ Fisher's Exact test $(p<0.05)$

There were also no significant differences in the time for eye opening, spontaneous ventilation, extubation, shaking hands and saying their complete names (Table III) in both groups.

Among postoperative complications, the incidence of nausea and pain was not statistically significant, although 4 patients in group 1 and 7 patients in group 2 referred nausea. The incidence of pain was identical in both groups (8 patients in each group). However, a statistically significant difference in the incidence of vomiting between the groups was observed, with eleven group 2 patients (55\%) presenting postoperative vomiting as compared to only one patient in group 1 (5\%) (Table IV).

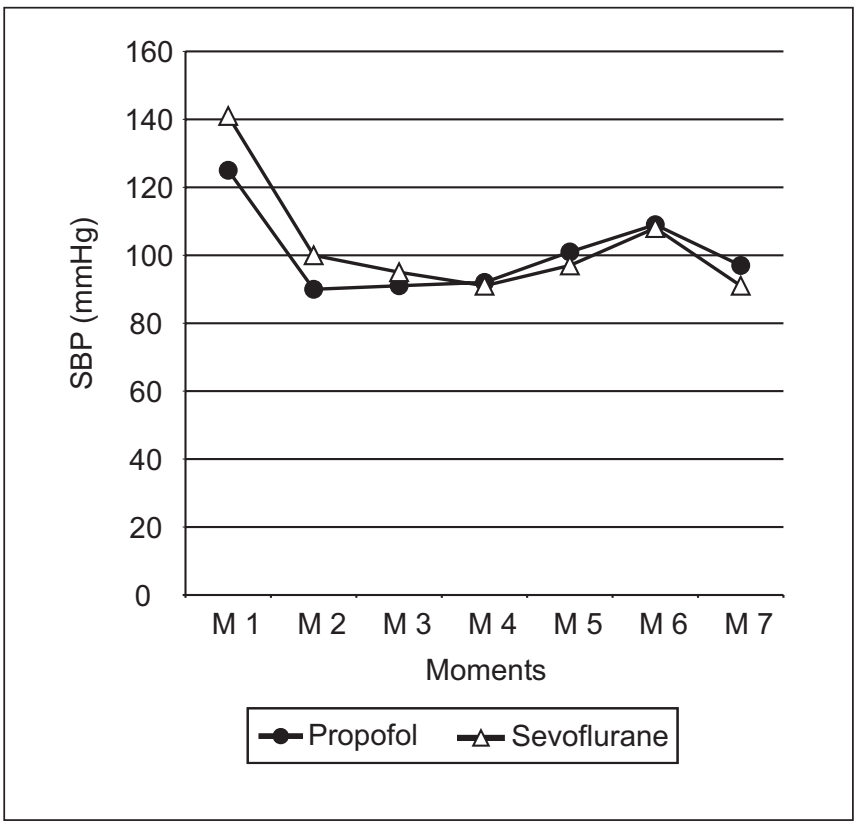

Figure 1 - Systolic Blood Pressure $(p>0.05)$

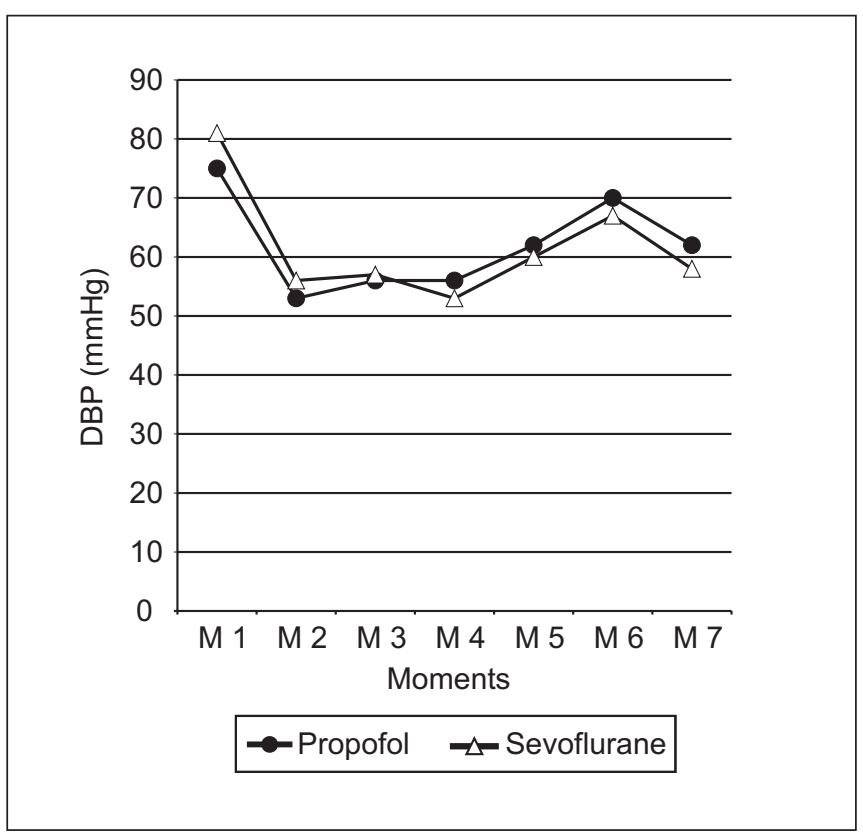

Figure 2 - Diastolic Blood Pressure $(p>0.05)$

Revista Brasileira de Anestesiologia Vol. 52, No 4, Julho - Agosto, 2002 


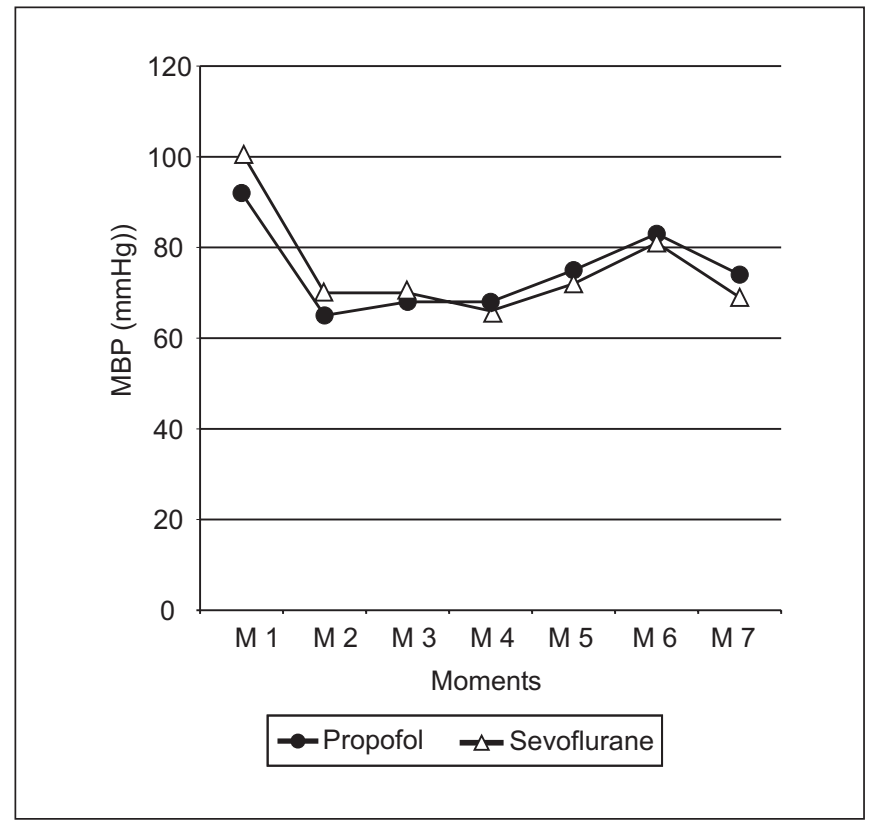

Figure 3 - Mean Blood Pressure $(p>0.05)$

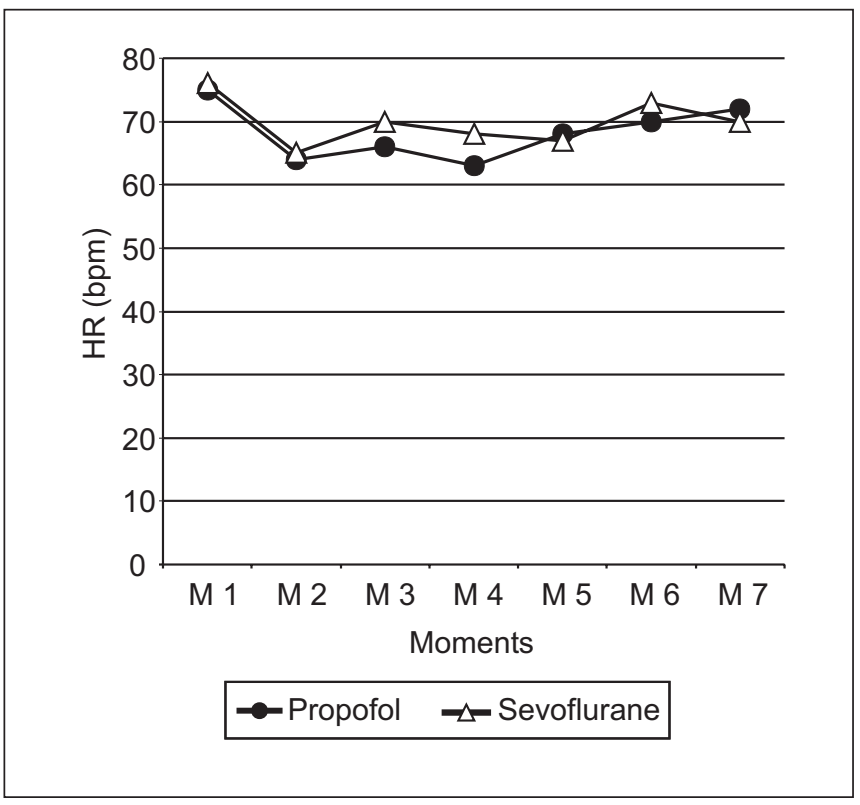

Figure 4 - Heart Rate $(p>0.05)$

Table III - Post-anesthetic Recovery (Mean \pm SD; in Minutes)

\begin{tabular}{lcc}
\hline & $\begin{array}{c}\text { Group 1 (Propofol) } \\
(\mathrm{n}=20)\end{array}$ & $\begin{array}{c}\text { Group 2 (Sevoflurane) } \\
(\mathrm{n}=20)\end{array}$ \\
\hline Eye openingA & $11,10 \pm 4,05$ & $14,39 \pm 6,10$ \\
Spontaneous ventilation & $11,10 \pm 4,27$ & $10,74 \pm 4,22$ \\
Extubation & $11,43 \pm 4,32$ & $13,92 \pm 5,36$ \\
Hand shaking & $12,50 \pm 4,06$ & $15,25 \pm 6,00$ \\
Say the name & $12,88 \pm 3,97$ & $16,04 \pm 4,65$ \\
\hline
\end{tabular}

Student's t test $(p>0.05)$

Revista Brasileira de Anestesiologia

Vol. 52, № 4, Julho - Agosto, 2002
Table IV - Postoperative Complications

\begin{tabular}{lcc}
\hline & $\begin{array}{c}\text { Group 1 (Propofol) } \\
(\mathrm{n}=20)\end{array}$ & $\begin{array}{c}\text { Group 2 (Sevoflurane) } \\
(\mathrm{n}=20)\end{array}$ \\
\hline Nausea & 4 & 7 \\
Vomiting & 1 & $11^{*}$ \\
Pain & 8 & 8 \\
\hline
\end{tabular}

* Fisher's Exact test $(p<0.05)$

\section{DISCUSSION}

This study has shown that both techniques had similar hemodynamic results in videolaparoscopic cholecystectomies, with a tendency to lowering blood pressure and heart rate in the studied moments. During anesthetic induction with propofol/remifentanil, hypotension and bradycardia severity depends on propofol infusion velocity and the opioid dose ${ }^{4,5}$. During sevoflurane induction, some authors ${ }^{6}$ refer less hypotension as compared to propofol. In our study, hypotension and bradycardia might was probably related to remifentanil infusion which, alone, has those dose-dependent para-effects ${ }^{7}$. Some studies ${ }^{8.9}$ report that the minimum propofol concentration needed to abolish eyelid reflex in most patients may be too high for elderly patients, thus promoting a greater BP decrease. There is a recommendation that remifentanil dose should be halved in patients above 65 years of age to minimize bradycardia and hypotension ${ }^{10}$.

Crystalloid infusion is indicated to prevent or treat initial arterial hypotension ${ }^{11}$. In our study, all patients received, before induction, $500 \mathrm{ml}$ of $0.9 \%$ saline solution. Previous studies have shown that propofol induction decrease blood pressure especially when associated to opioid infusion, which was also observed in our study, where $50 \%$ of the patients in group 1 have shown a significant decrease. This decrease was not associated to blood pressure normalization at tracheal intubation, showing the blockade of the hypertensive response at laryngoscopy, as described in a comparative study between sevoflurane-propofol and alfentanil ${ }^{12}$.

In spite of the relatively high incidence of hypotension in our study, this has been brief and easily controlled with vasopressors.

There were no statistically significant differences in the parameters used to evaluate anesthetic recovery (time for eye opening, spontaneous ventilation, extubation, hand shaking and saying the complete name), however, the propofol group showed shorter times. Jellish et al. ${ }^{13}$ refers earlier emergence with sevoflurane, due to its fast recovery profile provided by its poor solubility and less tissue accumulation. After long infusions, propofol elimination half-life is increased due to the accumulation of the drug in poorly vascularized peripheral tissues. It is possible that recovery times were similar in our study due to the faster propofol infusion and the non-accumulation of the drug ${ }^{13}$. Other studies ${ }^{6,14}$ have also shown a faster recovery with sevoflurane as compared to propofol, however without statistical significance.

No statistically significant differences were found in the incidence of nausea and pain but a higher incidence of vomiting 
was observed in the sevoflurane group. Some studies report a similar incidence of postoperative nausea and vomiting both in patients using sevoflurane and propofol ${ }^{13-15}$. Hwan et al. ${ }^{16}$ have shown results similar to ours, with less nausea and vomiting in the propofol group. Propofol has anti-emetic characteristics, even in surgeries causing nausea and vomiting 17 , such as cholecystectomies. Other factors contributing for those symptoms could be obesity, diabetes, young and female patients. In our study, the population was predominantly feminine in both groups. The incidence of postoperative vomiting in group 2 could be related to the use of opioids, if it was not used a fast eliminating opioid, such as remifentanil. Sevoflurane, for its agreeable odor and fast cognitive functions recovery, causes less nausea and vomiting than other inhalational agents, but it does not present propofol's anti-emetic characteristics.

The incidence of pain was similar in both groups. Some papers ${ }^{13,14}$ have reported the earlier need for analgesia in patients submitted to anesthesia with sevoflurane, although without statistically significant differences.

\section{CONCLUSIONS}

Data obtained in this study allow to conclude that: a) both techniques decrease blood pressure and heart rate; $b$ ) anesthetic recovery was similar for both techniques; c) there is a higher incidence of vomiting when remifentanil is associated to sevoflurane, as compared to propofol, in patients submitted to videolaparoscopic cholecystectomy.

\section{REFERÊNCIAS - REFERENCES}

01. White PF - Propofol, em: White PF - Tratado de Anestesia Venosa, $1^{\mathrm{a}}$ Ed, São Paulo, Artmed Editora Ltda, 2001;121-160.

02. Blobner M, Schneck HJ, Felber AR et al - Comparative study of the recovery phase: laparoscopic cholecystectomy following isoflurane, metohexital and propofol anesthesia. Anaesthesist, 1994;43:573-581.

03. Turazzi JC, Bedin A - Sevoflurano em cirurgia videolaparoscópica. Rev Bras Anestesiol, 1999;49:299-303.

04. Peacock JE, Lewis RP, Reilly CS et al - Effect of different rates of infusion of propofol for induction of anaesthesia in elderly patients. Br J Anaesth, 1990;65:346-352.

05. Billard V, Moulla F, Bourgain JL et al - Hemodynamic response to induction and intubation. Propofol/fentanyl interaction. Anesthesiology, 1994;81:1384-1393.

06. Thwaites A, Edmends S, Smith I - Inhalation induction with sevoflurane: a double-blind comparison with propofol. $\mathrm{Br} \mathrm{J}$ Anaesth, 1997;78:356-361.

07. Glass PS, Gan TJ, Howell S - A review of the pharmacokinetics and pharmacodynamics of remifentanil. Anesth Analg, 1999;89. S7-14.

08. Leslie K, Sessler DI, Smith WD et al - Prediction of movement during propofol/nitrous oxide anesthesia. Performance of concentration, electroencephalographic, pupilary, and hemodynamic indicators. Anesthesiology, 1996;84:52-63.
09. Vuyk J, Engbers FH, Burm AGL et al - Pharmacodynamic interaction between propofol e alfentanil when given for induction of anesthesia. Anesthesiology, 1996;84:288-299.

10. Grounds RM, Morgam M, Lumley J - Some studies on the properties of the intravenous anesthetic, propofol a review. Postgrad Med J, 1985;61:(Suppl 3);90-95.

11. Camu F, Roystron D - Inpatient experience with remifentanil. Anesth Analg, 1999;89:S15-21.

12. Nathan N, Vial G, Benrhaiem M et al - Induction with propofol target-concentration infusions vs $8 \%$ sevoflurane inhalation and alfentanil in hypertensive patients. Anaesthesia, 2001;56: 248-271.

13. Jellish WS, Lien CA, Fontenot $\mathrm{HJ}$ et al - The comparative effects of sevoflurane versus propofol in the induction and maintenance of anesthesia in adult patients. Anesth Analg, 1996;82:479-485.

14. Watson KR, Shah MV - Clinical comparison of "single agent" anaesthesia with sevoflurane versus target controlled infusion of propofol. Br J Anaesth, 2000;85:541-546.

15. Rowbotham DJ, Peacock JE, Jones RM et al - Comparison of remifentanil in combination with isoflurane or propofol for short-stay surgical procedures. Br J Anaesth, 1998;80:752-755.

16. Joo HS, Perks WJ - Sevoflurane versus propofol for anesthetic induction: a meta-analysis. Anesth Analg, 2000;91:213-219.

17. Mc Collum JS, Milligan KR, Dundee JN - The antiemetic action of propofol. Anaesthesia, 1988;43:239-240.

\section{RESUMEN}

Estivalet FF, Bagatini A, Gomes CR - Remifentanil Asociado al Propofol o Sevoflurano para Colecistectomia Videolaparoscópica. Estudio Comparativo

Justificativa y Objetivos - Técnicas de anestesia venosa, inhalatoria o combinadas han sido utilizadas para colecistectomias por videolaparoscopia. El objetivo de este estudio fue comparar la utilización de remifentanil asociado al propofol o sevoflurano con relación a los parámetros hemodinámicos, la recuperación de la anestesia y a los efectos colaterales.

Método - Fueron estudiados 40 pacientes, divididos en 2 grupos: Grupo 1 (G1) - Inducción con propofol en concentración plasmática alvo-controlada de $3 \mu \mathrm{g} . \mathrm{ml}^{-1}$ y remifentanil en la dosis de $0,3 \mu \mathrm{g} \cdot \mathrm{kg}^{-1} \cdot \mathrm{min}^{-1}$ en bomba de infusión. Atracúrio $\left(0,5 \mathrm{mg} \cdot \mathrm{kg}^{-1}\right)$ fue administrado para la intubación traqueal. La anestesia fue mantenida con propofol en infusión alvo-controlada de 2,5 a $3 \mu \mathrm{g} \cdot \mathrm{ml}^{-1}$ y oxígeno a $100 \%$, y remifentanil en infusión continua $\left(0,3 \mu \mathrm{g} \cdot \mathrm{kg}^{-1} \cdot \mathrm{min}^{-1}\right)$. Grupo 2 (G2) - Inducción con sevoflurano en la concentración de $6 \%$ y oxígeno a $100 \%$ con flujo de 4 L. min $^{-1}$ y remifentanil en la dosis de $0,3 \mu \mathrm{g} \cdot \mathrm{kg}^{-1}$. $\mathrm{min}^{-1}$, en bomba de infusión. Después de la perdida de la consciencia, la concentración de sevoflurano fue reducida para $2 \%$ con flujo de oxígeno de 2 L. $\mathrm{min}^{-1}$; de la misma forma que el G1, fue administrado atracúrio en la dosis de $0,5 \mathrm{mg} \cdot \mathrm{kg}^{-1}$. La infusión de remifentanil fue mantenida durante toda la cirugía en la dosis de $0,3 \mu \mathrm{g} \cdot \mathrm{kg}^{-1} . \mathrm{min}^{-1}$. La media de la frecuencia cardíaca (FC), y de las presiones arteriales sistólicas (PAS), diastólica (PAD) y media (PAM) fueron medidas en los siguientes momentos: M1, antes de la inducción anestésica; M2, 1 minuto antes de la IOT; M3, 1 minuto después IOT; M4, 5 minutos después IOT; M5, 5 minutos después de la incisión quirúrgica; M6 y M7,10 y 30 minutos, respectivamente, después el pneumoperitonio. Después del término del procedimiento fueron analizados los tiempos, en minutos, para abertura ocular, ventilación espontanea, extubación, hablar el nombre completo y el aprieto de la mano del anestesiologista, sobre comando. 
REMIFENTANIL ASSOCIATED TO PROPOFOL OR SEVOFLURANE FOR

VIDEOLAPAROSCOPIC CHOLECYSTECTOMY. A COMPARATIVE STUDY

También fueron estudiada la frecuencia del uso de atropina y efedrina y la ocurrencia de náuseas, vómitos y dolor.

Resultados - Las alteraciones hemodinámicas (PAS, PAD,

PAM y FC) y el despertar de los pacientes no tuvieron diferencias significativas entre los grupos. El uso de efedrina y atropina fueron semejantes. Entre las complicaciones solamente los vómitos tuvieron mayor incidencia en el Grupo 2.
Conclusiones - Ambas técnicas promovieron diminución de la presión arterial y de la frecuencia cardíaca. La recuperación anestésica es semejante para las dos técnicas. La incidencia de vómitos es mayor cuando el remifentanil fue asociado al sevoflurano. 